\title{
Dynamics, Control and Planning for Cooperative Manipulation of Payloads Suspended by Cables from Multiple Quadrotor Robots
}

\author{
Koushil Sreenath \\ Department of Mechanical Engineering \\ and Applied Mechanics, University of Pennsylvania \\ Philadelphia, PA 19104 \\ Email: koushils@seas.upenn.edu
}

\author{
Vijay Kumar \\ Department of Mechanical Engineering \\ and Applied Mechanics, University of Pennsylvania \\ Philadelphia, PA 19104 \\ Email: kumar@seas.upenn.edu
}

\begin{abstract}
We address the problem of cooperative transportation of a cable-suspended payload by multiple quadrotors. In previous work, quasi-static models have been used to study this problem. However, these approaches are severely limited because they ignore the payload dynamics, and do not explicitly model the underactuation in the control problem. Thus, there are no guarantees on the payload trajectory or the cable tensions, which must be non negative. In this paper, we develop a complete dynamic model for the case when payload is a point load and for the case when the payload is a rigid body. We show in both cases the resulting system is differentially flat when the cable tensions are strictly positive. We also consider the case where the tensions are non negative (including the case with zero tensions) and establish that these systems are differentially flat hybrid systems by considering the switching dynamics induced by the unilateral tension constraints. We use the differential flatness property to find dynamically feasible trajectories for the payload+quadrotors system. We show using numerical and experimental methods that these trajectories are superior to those obtained by quasi-static models.
\end{abstract}

\section{INTRODUCTION}

Aerial robotics is a growing field with a wide range of civil and military applications. The last five years have seen the maturation of micro aerial vehicles, especially quadrotors, that range from tens of centimeters to several meters with payloads that are limited to less than several kilograms [1]. While these robots can maneuver in highly-constrained, threedimensional environments, they are limited in terms of their payload carrying capacity. However, these robots can carry payloads beyond the capacity of individuals by collaborating in manipulation and transportation tasks. Teams of robots can be used for transportation in search and rescue missions, environmental monitoring, and for surveillance tasks.

In this paper, we are particularly interested in cooperative transportation tasks where the payload is suspended by cables from multiple quadrotors. This task is closely related to aerial towing, the manipulation of a payload suspended by a cable from a moving aerial robot [2]. A single quadrotor with a cable suspended load has been studied in [3]. Cooperative aerial towing has also been studied, in particular by [4, 5, 6, 7].

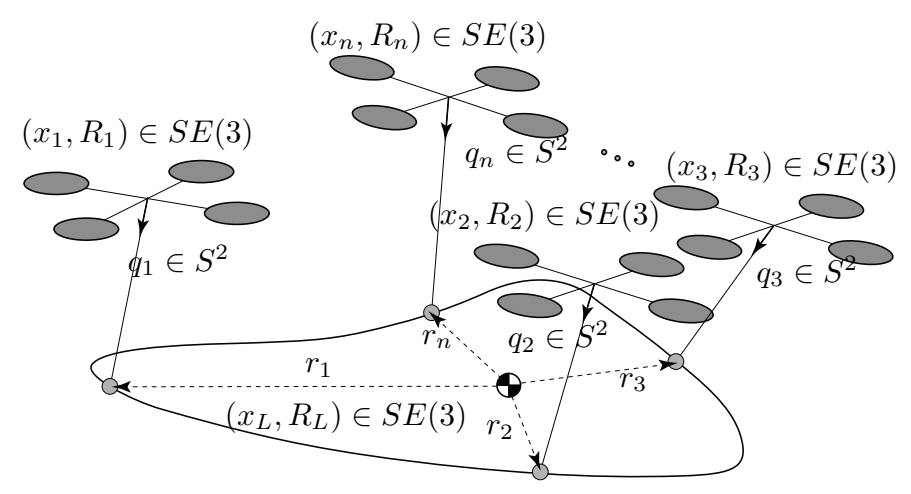

Fig. 1: Load being transported by $\mathrm{n}$ quadrotors.

In all these cases, equilibrium position and orientation of the suspended payload are configurations in which the gravity wrench is equilibrated by the wrenches exerted by the cables. From a static analysis, it is clear that at least three cables are required to suspend the payload in any desired configuration. Indeed this case has been studied in detail by [4, 5]. However, all these approaches are based on quasi-static models, with the assumption that the load (and therefore the quadrotors) have motions that give rise to negligible inertial forces. However, as seen in the experimental results and videos in these papers, it is quite clear that this quasi-static assumption is not valid. Indeed it is impossible to make any assertions about the resulting trajectory without explicitly modeling and analyzing the full dynamics of the system.

We address this limitation in all previous papers by studying the dynamics of cooperative manipulation by using a complete dynamic model for the cases when payload is (a) a point load; and (b) a three-dimensional rigid body. We show in both cases the resulting system is differentially flat when the cable tensions are strictly positive. We also consider the case where the tensions are non negative (including the case with zero tensions) and establish that these systems are differentially flat hybrid systems by considering the switching dynamics induced by the unilateral tension constraints. We use the differential 
flatness property to find dynamically feasible trajectories for the payload+quadrotors system. We show using numerical and experimental methods that these trajectories are superior to those obtained by quasi-static models.

The rest of the paper is structured as follows. Section [I establishes the differential-flatness of the $n$-quadrotor cablesuspended load system, both with point-mass and rigid-body loads. Section III presents the hybrid model for both these systems, and establishes that these are differentially-flat hybrid systems. Section IV presents numerical and experimental results for a rigid-body load carried by three quadrotors. Finally Section $\nabla$ provides concluding remarks and thoughts on future work.

\section{DifFERENTIAL FLATNESS}

We will consider two systems, a point-mass load suspended by cables from $n$ quadrotors, and a rigid-body load also suspended by cables from $n$ quadrotors. To enable planning dynamic trajectories of the cable-suspended load for aerial transportation, we will demonstrate that both these systems are differentially flat $[8,9,2]$. Differential-flatness has been employed for planning dynamic trajectories for quadrotor systems [10]. In Section [IV] we will make use the flat outputs to plan dynamic trajectories.

Definition 1. Differentially-flat system [9]: A system $\dot{x}=$ $f(x, u), x \in \mathbb{R}^{n}, u \in \mathbb{R}^{m}$, is differentially flat if there exists outputs $y \in \mathbb{R}^{m}$ of the form $y=y\left(x, u, \dot{u}, \cdots, u^{(p)}\right)$, such that the states and the inputs can be expressed as $x=x\left(y, \dot{y}, \cdots, y^{(q)}\right), u=u\left(y, \dot{y}, \cdots, y^{(q)}\right)$, where $p, q$ are finite integers.

To demonstrate the differential flatness property of the multiple-quadrotor cable-suspended system, we first develop a dynamical model of system based on Newton-Euler equations, and then use this to identify a set of flat outputs.

For the two systems presented in this paper, we will make the following assumptions,

1) Cables are massless and do not stretch.

2) Cables are attached at the quadrotor's center of mass.

3) Air drag on the quadrotors and the load is negligible.

4) When a cable goes from being slack to taut, there is a discrete change in the velocity of the system, and this is modeled based on a perfectly inelastic collision.

\section{A. Point-Mass Load}

We first consider the point-mass load suspended by $n \geq 1$ quadrotors as shown in Figure 2 The independent degrees of freedom (DOF) of this system are the load position, $x_{L} \in \mathbb{R}^{3}$, the attitude of the suspended cables, $q_{i} \in S^{2}$, and the attitude of the quadrotors, $R_{i} \in S O(3)$ (See Table \ for definitions of various symbols used in the paper.) Defining the length of the $i^{t h}$ cable as $L_{i}$, from the geometry of how the load is attached to the quadrotors, we have the quadrotor position, $x_{i} \in \mathbb{R}^{3}$, given by the following kinematic relation,

$$
x_{i}=x_{L}-L_{i} q_{i} .
$$

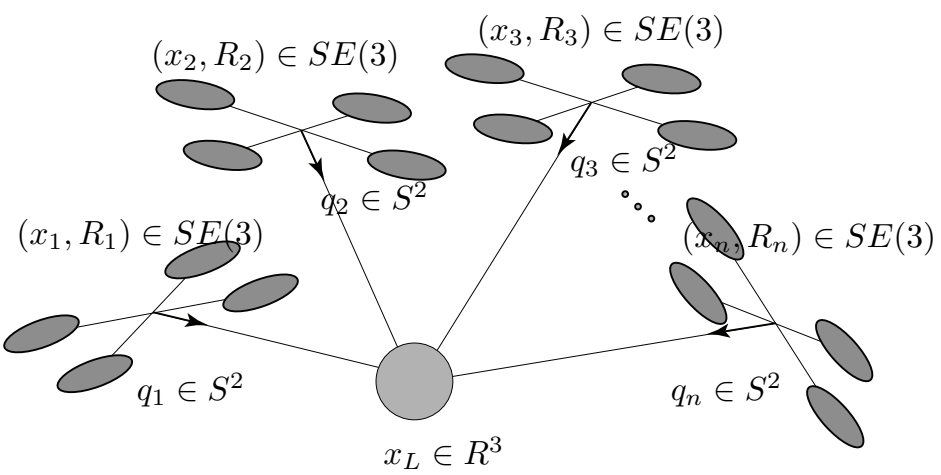

Fig. 2: Point-Mass Load being transported by $\mathrm{n}$ quadrotors.

Using the tension in the cables, $T_{i} \in \mathbb{R}$, the Euler dynamics of the $n$ quadrotors and the load can be easily written down as follows,

$$
\begin{gathered}
m_{i} \ddot{x}_{i}=f_{i} R_{i} e_{3}-m_{i} g e_{3}+T_{i} q_{i}, \\
J_{i} \dot{\Omega}_{i}+\Omega_{i} \times J_{i} \Omega_{i}=M_{i}, \\
m_{L} \ddot{x}_{L}=-\sum T_{i} q_{i}-m_{L} g e_{3},
\end{gathered}
$$

where $m_{i}, J_{i}, f_{i}$ are the mass, inertia and thrust of the $i^{t h}$ quadrotor, $m_{L}$ is mass of the load, and $e_{3}$ is the standard unit vector along the $\mathrm{z}$-axis of the world.

Lemma 1. (Differential-Flatness of the $n$ quadrotor, pointmass load system, $n \geq 1$.) $\mathcal{Y}_{n}=\left(x_{L}, T_{i} q_{i}, \psi_{j}\right)$, for $i \in$ $\{2, \cdots, n\}, j \in\{1, \cdots, n\}$ is a set of flat outputs for the $n$ quadrotor, point-mass load system, where $\psi_{j}$ is the yaw angle of the $j^{\text {th }}$ quadrotor.

Proof: From $x_{L}$ and its higher order derivatives, the left hand side of (4) can be determined. Next, from the knowledge of the flat outputs $T_{i} q_{i}$, for $i \in\{2, \cdots, n\}, T_{1} q_{1}$ can be determined from (4). The unit vectors $q_{i}=T_{i} q_{i} /\left\|T_{i} q_{i}\right\|$, and the tension $T_{i}=T_{i} q_{i} \cdot q_{i}$ can also be determined for $i \in\{1, \cdots, n\}$. The quadrotor positions can then be determined using (1). All remaining quantities, $R_{i}, \Omega_{i}, f_{i}, M_{i}$ can be determined from knowledge of $x_{i}, \psi_{i}$ and their higher-order derivatives, since $\left(x_{i}, \psi_{i}\right)$ are flat outputs for a quadrotor.

Remark 1. The load position, $x_{L}$ needs to be differentiated six times, and the tensions $T_{i} q_{i}, i>2$ needs to be differentiated four times to obtain the entire state of the system, along with the feedforward thrusts and moments for the quadrotors.

\section{B. Rigid-Body Load}

Having established the differential-flatness of the $n$ quadrotor, point-mass load, we now consider a rigid body load. First, from the geometry of how the load is attached to the quadrotors, see Figure 1 we have the quadrotor position given by the following kinematic relation,

$$
x_{i}=x_{L}+R_{L}\left(r_{i}-L_{i} q_{i}\right),
$$

where $x_{L}$ is the position of the load, $x_{i}$ the position of the $\mathrm{i}^{\text {th }}$ quadrotor, $R_{L}$ the orientation of the load, $q_{i}$ the unit vector from the $\mathrm{i}^{\text {th }}$ quadrotor to the attachment point on the load 


\begin{tabular}{ll}
$m_{L} \in \mathbb{R}$ & Mass of Load \\
$J_{L} \in \mathbb{R}^{3 \times 3}$ & Inertia matrix of the load with respect to the body-fixed frame \\
$R_{L} \in S O(3)$ & The rotation matrix of the load from the body-fixed frame to the inertial frame \\
$\Omega_{L} \in \mathbb{R}^{3}$ & Angular velocity of the load in the body-fixed frame \\
$x_{L}, v_{L} \in \mathbb{R}^{3}$ & Position and velocity vectors of the center of mass of the load in the inertial frame \\
\hline$m_{i} \in \mathbb{R}$ & Mass of $i^{t h}$ quadrotor \\
$J_{i} \in \mathbb{R}^{3 \times 3}$ & Inertia matrix of the $i^{\text {th }}$ quadrotor with respect to the body-fixed frame \\
$R_{i} \in S O(3)$ & The rotation matrix of the $i^{t h}$ quadrotor from the body-fixed frame to the inertial frame \\
$\Omega_{i} \in \mathbb{R}^{3}$ & Angular velocity of the $i^{\text {th }}$ quadrotor in the body-fixed frame \\
$x_{i}, v_{i} \in \mathbb{R}^{3}$ & Position and velocity vectors of the center of mass of the $i^{t h}$ quadrotor in the inertial frame \\
$f_{i} \in \mathbb{R}$ & Thrust produced by the $i^{t h}$ quadrotor \\
$M_{i} \in \mathbb{R}^{3}$ & Moment produced by the $i^{t h}$ quadrotor \\
$\psi_{i} \in \mathbb{R}$ & Yaw angle of the $i^{t h}$ quadrotor \\
\hline$q_{i} \in S^{2}$ & Unit vector from the $i^{t h}$ quadrotor to its attachment point on the load in body-fixed frame of the load \\
$r_{i} \in \mathbb{R}^{3}$ & Vector form the center of mass of the load to the attachment point of the $i^{t h}$ quadrotor to the load \\
$L_{i} \in \mathbb{R}_{i}$ & Length of the cable between the $i^{t h}$ quadrotor and the load \\
$T_{i} \in \mathbb{R}$ & Tension in the the cable between the $i^{t h}$ quadrotor and the load \\
$e_{1}, e_{2}, e_{3} \in \mathbb{R}^{3}$ & Standard unit vector along $x, y, z$ axes in the world frame
\end{tabular}

TABLE I: Various symbols being used.

expressed in the body-fixed frame of the load, and $r_{i}$ the vector from the center-of-mass of the load to the attachment point in the body-fixed frame of the load.

Using the tension in the cables, $T_{i}$, the Euler dynamics of the $n$ quadrotors and the rigid-body load can be easily written down as follows,

$$
\begin{gathered}
m_{i} \ddot{x}_{i}=f R_{i} e_{3}-m_{i} g e_{3}+R_{L} T_{i} q_{i}, \\
J_{i} \dot{\Omega}_{i}+\Omega_{i} \times J_{i} \Omega_{i}=M_{i}, \\
m_{L} \ddot{x}_{L}=-\sum R_{L} T_{i} q_{i}-m_{L} g e_{3}, \\
J_{L} \dot{\Omega}_{L}+\Omega_{L} \times J_{L} \Omega_{L}=\sum r_{i} \times-T_{i} q_{i},
\end{gathered}
$$

where $i \in\{1, \cdots, n\}$, and all other symbols are as defined in Table [

Lemma 2. (Differential-Flatness of the $n$ quadrotor, rigidbody load system, $n \geq 3$.) $\mathcal{Y}_{n}=\left(x_{L}, R_{L}, \Lambda, \psi_{j}\right)$ for $j \in$ $\{1, \cdots, n\}$ is a set of flat outputs for the $n$ quadrotor, rigidbody load system, where $\Lambda \in \mathbb{R}^{3 n-6}$ satisfies,

$$
\underline{T}=\Phi^{+} W+N \Lambda,
$$

with $\underline{T}, W$ defined as

$$
\underline{T}=\left[\begin{array}{c}
T_{1} q_{1} \\
T_{2} q_{2} \\
\vdots \\
T_{n} q_{n},
\end{array}\right], \quad W=-\left[\begin{array}{c}
R_{L}^{T}\left(m_{L}\left(\ddot{x}_{L}+g e_{3}\right)\right) \\
J_{L} \dot{\Omega}_{L}+\Omega_{L} \times J_{L} \Omega_{L}
\end{array}\right],
$$

where $\Phi^{+}, N$ are respectively the Moore-Penrose generalized inverse and the kernel of

$$
\Phi=\left[\begin{array}{cccc}
I & I & \cdots & I \\
\hat{r}_{1} & \hat{r}_{2} & \cdots & \hat{r}_{n}
\end{array}\right]
$$

with the hat map $\therefore: \mathbb{R}^{3} \rightarrow$ so(3) defined by the condition that $\hat{x} y=x \times y$, for all $x, y \in \mathbb{R}^{3}$.

Proof: Notice that (8), (9) can be written as

$$
-\left[\begin{array}{c}
R_{L}^{T}\left(m_{L}\left(\ddot{x}_{L}+g e_{3}\right)\right) \\
J_{L} \dot{\Omega}_{L}+\Omega_{L} \times J_{L} \Omega_{L}
\end{array}\right]=\Phi\left[\begin{array}{c}
T_{1} q_{1} \\
T_{2} q_{2} \\
\vdots \\
T_{n} q_{n}
\end{array}\right] .
$$

From (11), we can denote the LHS of (13) by,

$$
W=\left[\begin{array}{c}
W_{f} \\
W_{M}
\end{array}\right] \text {. }
$$

This is, in effect, the load wrench consisting of the net force and moment that is produced by the tensions. Further from

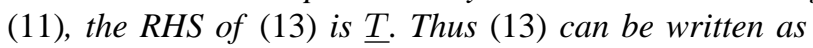

$$
\Phi \underline{T}=W,
$$

which is an under-determined set of equations with the general solution given by (10). Note that $N$ is a $3 n \times(3 n-6)$ matrix whose columns span the kernel of $\Phi$, representing the constraints on the internal forces in the system.

From the flat outputs $\left(x_{L}, R_{L}\right)$ and their higher-order derivatives, $W$ can be determined from (11). Further from the flat output $\Lambda, T_{i} q_{i}$ can be determined for $i \in\{1, \cdots, n\}$ through (10). Then, the unit vector $q_{i}=T_{i} q_{i} /\left\|T_{i} q_{i}\right\|$, and the tension $T_{i}=T_{i} q_{i} \cdot q_{i}$ can also be determined. The quadrotor positions can then be determined using (5). All remaining quantities, $R_{i}, \Omega_{i}, f_{i}, M_{i}$ can be determined from knowledge of $x_{i}, \psi_{i}$ and their higher-order derivatives, since $\left(x_{i}, \psi_{i}\right)$ are flat outputs for a quadrotor.

Remark 2. The load position, $x_{L}$ needs to be differentiated six times, and the load orientation $R_{L}$ and the mapped tensions $\Lambda$ need to be differentiated four times to obtain the entire state of the system, along with the feedforward thrusts and moments for the quadrotor.

Remark 3. When the anchor points are symmetric about the center-of-mass of the load, i.e., when

$$
\Sigma r_{i}=0,
$$

(10) can be simplified to

$$
\underline{T}=\left[\begin{array}{c}
\frac{1}{n} W_{f}+\Pi^{-1}\left(W_{M} \times r_{1}\right) \\
\vdots \\
\frac{1}{n} W_{f}+\Pi^{-1}\left(W_{M} \times r_{n}\right)
\end{array}\right]+N \Lambda,
$$

where

$$
\Pi=\left[-\sum_{i=1}^{n} \hat{r}_{i} \hat{r}_{i}\right]
$$


is the second moment of distribution of the anchor points.

Remark 4. We can derive a special basis for $N$ by representing the internal forces by pairs of equal and opposite forces. Define by $u_{i j}$ the unit vector from anchor point $i$ to $j$, i.e.,

$$
u_{i j}=\frac{r_{j}-r_{i}}{\left\|r_{j}-r_{i}\right\|}
$$

The columns of $N$ can then be chosen as,

$$
\left[\begin{array}{lllllll}
0 & \cdots & u_{i j} & \cdots & -u_{i j} & \cdots & 0
\end{array}\right]^{T},
$$

where only the $i^{\text {th }} 3 \times 1$ component and the $j^{\text {th }} 3 \times 1$ components are non-zero. For example, for $n=3$, we have,

$$
N_{9 \times 3}=\left[\begin{array}{ccc}
u_{12} & u_{13} & 0 \\
-u_{12} & 0 & u_{23} \\
0 & -u_{13} & -u_{23}
\end{array}\right] \text {, }
$$

and,

$$
\Lambda=\left[\begin{array}{l}
T_{12} \\
T_{13} \\
T_{23}
\end{array}\right] .
$$

Under this basis, a good choice for $\Lambda(t)$ would be $\Lambda \equiv 0$, which would ensure that the tensions in the cables have no components along $u_{i j}$, thereby resulting in the tension not performing any isometric work.

Remark 5. An alternative choice for $\Lambda$ would be

$$
\Lambda=\left[\begin{array}{c}
T_{1} q_{1} \cdot e_{1} \\
T_{1} q_{1} \cdot e_{2} \\
T_{2} q_{2} \cdot e_{1} \\
T_{j} q_{j}
\end{array}\right], \quad 3<j \leq n .
$$

Note that there exists a diffeomorphism between the flat output space and the state space. This implies that any motion that can be generated through one set of flat output variables can also be generated through another choice of the flat output variables. The choice of flat output variables does not affect the system motion, although some choices may be easier to use than others for designing the trajectories and the feedforward control.

Remark 6. For the 2-quadrotor load carrying system, $(n=2)$, $\mathcal{Y}_{2}=\left(x_{L}, R_{L}, \psi_{k}\right)$ for $k \in\{1,2\}$ does not form a set of flat outputs, since there exists a degree of underactuation corresponding to rotation about the line joining the two contact points that can not be determined from the flat outputs. In particular, for $n=2$, one would expect (13) to be a set of six equations in six variables, however, for this case, $\Phi \in \mathbb{R}^{6 \times 6}$ is rank-deficient for all $r_{1}, r_{2}$.

Remark 7. The flat output $\Lambda$ can be so chosen such that $T_{i}(t)>0, \forall t$.

Table II contains a summary of the key results in this section. It includes the number of degrees of freedom, number degrees of underactuation, and the flat outputs for the pointmass load with $n$ quadrotors including the special case of $n=1$, and for the rigid-body load with $n$ quadrotors, including the special case of $n=3$.

\section{HybRID SYSTEM MOdEL}

In the previous section, we developed the dynamics and established that the $n$ quadrotor system with either a cable-suspended point-mass load or a rigid-body load are differentially-flat. Now we explicitly consider the case when the tension in any of the cables drops to zero.

If the tension in any of the cables goes to zero, or if tension in any of the slack cables is reestablished, then the system dynamics switches, making this a hybrid system. Without loss of generality, we can assume that at most one cable tension can either drop to zero or one slack cable can get its tension reestablished to a nonzero value at any given moment. Moreover, we can also assume that this happens sequentially 1 , i.e., starting with all $n$ cables in tension, only the $n^{\text {th }}$ cable tension can drop to zero, and following this either the $(n-1)^{t h}$ cable tension can drop to zero or the $n^{\text {th }}$ cable tension can get reestablished, and so on as illustrated in Figure 3 . We denote by $\Sigma_{k}$ the continuous-time system that has slack cables for all quadrotors with indices greater than $k$, i.e., $T_{i} \equiv 0, i>k$.

Furthermore, we will model the discrete transition map from $\Sigma_{k}$ to $\Sigma_{k-1}$, that occurs when a cable tension drops to zero, as the identity map, and also enforce the tension $T_{i} \equiv 0, i>k$. We will model the discrete transition map $\Delta_{k}$ from $\Sigma_{k-1}$ to $\Sigma_{k}$ that occurs when a tension is reestablished as an inelastic collision (see Assumption 4), resulting in a discrete change in velocity. Moreover, we will assume $\Delta_{k}$ is a smooth map. The dynamics of the system $\Sigma_{k}$ for the point-mass load is as below

$$
\Sigma_{k}:\left\{\begin{array}{cr}
m_{i} \ddot{x}_{i}=f_{i} R_{i} e_{3}-m_{i} g e_{3}+T_{i} q_{i}, & X \notin S_{k}, \\
J_{i} \dot{\Omega}_{i}+\Omega_{i} \times J_{i} \Omega_{i}=M_{i}, & T_{i} \equiv 0, i>k \\
m_{L} \ddot{x}_{L}=-\sum T_{i} q_{i}-m_{L} g e_{3}, & \\
X^{+}=\Delta_{k+1}\left(X^{-}\right), & X \in S_{k}
\end{array}\right.
$$

where $X$ is the state of the entire system, and

$$
S_{k}=\left\{X \mid\left\|x_{k}-x_{L}\right\|=L_{k}, \frac{d}{d t}\left\|x_{k}-x_{L}\right\|>0\right\}
$$

defines the guard (using hybrid system terminology from [11]) when the distance between the $k^{\text {th }}$ quadrotor and its attachment point to the load reaches the length of the cable.

We will next demonstrate that the hybrid system under consideration is a differentially-flat hybrid system, as defined below.

Definition 2. A differentially-flat hybrid system is a hybrid system where each subsystem is differentially-flat, with the guards being functions of the flat outputs and their derivatives, and moreover there are sufficiently smooth transition maps from the flat output space of one subsystem to the flat output space of the subsequent subsystem.

Remark 8. A differentially-flat hybrid system does not imply all the states and inputs can be obtained by differentiating a set

\footnotetext{
${ }^{1}$ For inelastic collisions (Assumption 4), the results do not depend on the order in which these transitions occur.
} 


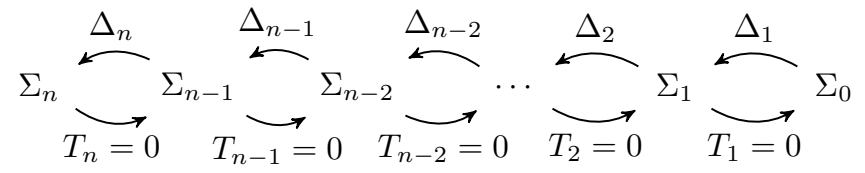

Fig. 3: Transition between subsystems as tension in the cable drops to zero or is reestablished to a positive value.

of smooth flat outputs. After all, the system is hybrid, and we expect discrete jumps in states and possibly inputs. Instead, we mean that each subsystem is differentially flat, and that the flat outputs of a subsequent subsystem arise as smooth functions of the flat outputs of the current subsystem, mapped through the transition map between the two subsystems.

Theorem 1. The multiple-quadrotor cable-suspended pointmass load is a differentially flat hybrid system for $n \geq 1$.

Proof: Suppose $\mathcal{Z}_{n}=\mathcal{Y}_{n}$ is a set of flat outputs for the $n$ quadrotor, point-mass load system from Lemma 1 Now suppose at some event, the tension in the cable for one of the quadrotors becomes zero. Since the system is differentially flat, this event is known from $\mathcal{Z}_{n}$ and its derivatives. Moreover, the new system $\Sigma_{n-1}$ with $n-1$ quadrotors and load, and with a single free quadrotor is also differentially flat since the $n-1$ quadrotor load system is differentially flat by Lemma 1 and the single quadrotor is also differentially flat. Moreover, the the transition map transforms the flat outputs $\mathcal{Z}_{n}$ to $\mathcal{Z}_{n-1}=$ $\left[\begin{array}{ll}\mathcal{Y}_{n-1} & \mathcal{Y}_{0}^{n}\end{array}\right]$, where $\mathcal{Y}_{n-1}$ corresponds to the flat output of the $n-1$ quadrotors and load system, and $\mathcal{Y}_{0}^{n}$ corresponds to the flat output of the $n^{\text {th }}$ quadrotor.

When the tension gets re-established, we can obtain the initial value of $\mathcal{Z}_{n}$ by mapping the flat output $\mathcal{Z}_{n-1}$ and its higher-order derivatives through the transition map $\Delta_{n}$.

We can sequentially compose this all the way to having all the tensions going to zero, and the load undergoing ballistic motion.

Next, we consider the multiple-quadrotor system with a rigid-body payload and demonstrate that this system is also a differentially-flat hybrid system. The hybrid dynamics of this system are given as,

$$
\Sigma_{k}:\left\{\begin{array}{c}
m_{i} \ddot{x}_{i}=f R_{i} e_{3}-m_{i} g e_{3}+R_{L} T_{i} q_{i}, \\
J_{i} \dot{\Omega}_{i}+\Omega_{i} \times J_{i} \Omega_{i}=M_{i}, \\
m_{L} \ddot{x}_{L}=-\sum R_{L} T_{i} q_{i}-m_{L} g e_{3}, \quad T_{i} \equiv 0, i>k \\
J_{L} \dot{\Omega}_{L}+\Omega_{L} \times J_{L} \Omega_{L}=\sum r_{i} \times-T_{i} q_{i}, \\
X^{+}=\Delta_{k}\left(X^{-}\right),
\end{array}\right.
$$

Theorem 2. The multiple-quadrotor with a cable-suspended rigid-body load is a differentially flat hybrid system for $n>3$.

Proof: The proof follows in a similar way to the previous theorem for $n>3$. For $n=3$, the tension in the cable attached

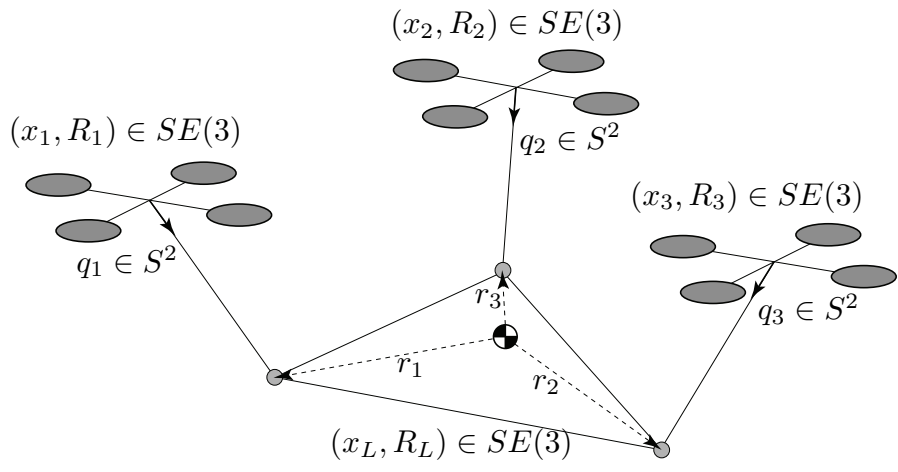

Fig. 4: Load being transported by three quadrotors.

to Quadrotor 3 drops to zero, and the resulting system is no longer differentially flat by Remark 6

\section{RESUlts}

Having established the differential-flatness of the $n$ quadrotor, cable-suspended point-mass and rigid-body load systems, we will demonstrate numerical and experimental results for the rigid-body case with $n=3$ quadrotors. An illustration of this is as shown in Figure 4.

\section{A. Numerical Results}

We choose the flat outputs for the $n=3$ quadrotor system with rigid-body load as,

$$
\mathcal{Y}_{3}=\left[\begin{array}{c}
x_{L} \\
R_{L} \\
T_{1} q_{1} \cdot e_{1} \\
T_{1} q_{1} \cdot e_{2} \\
T_{2} q_{2} \cdot e_{1} \\
\psi_{1} \\
\psi_{2} \\
\psi_{3}
\end{array}\right]
$$

Following [10], we could plan a dynamic trajectory that minimizes the $6^{\text {th }}$ derivative of the load position, leading to a minimum snap trajectory for the quadrotors. However, we consider instead a simple trajectory that serves to illustrate the choice of flat outputs and planning in flat space. The trajectory for the load is chosen to be an ellipse in the $y-z$ plane with the frequency $f$, given by,

$$
x_{L}(t)=\left[\begin{array}{c}
0 \\
A_{y} \cos (2 \pi f t) \\
A_{z} \sin (2 \pi f t)
\end{array}\right]
$$

The other flat outputs are chosen as follows,

$$
\begin{aligned}
R_{L}(t) & \equiv I, \\
T_{1}(t) q_{1}(t) \cdot e_{1} & \equiv(1 / 3) R_{y}(\pi / 6) m_{L} g \cdot e_{1} \\
T_{1}(t) q_{1}(t) \cdot e_{2} & \equiv(1 / 3) R_{y}(\pi / 6) m_{L} g \cdot e_{2} \\
T_{2}(t) q_{2}(t) \cdot e_{2} & \equiv(1 / 3) R_{y}(-\pi / 6) m_{L} g \cdot e_{2} \\
\psi_{k}(t) & \equiv 0, \quad k \in\{1,2,3\} .
\end{aligned}
$$




\begin{tabular}{|c|c|c|c|c|c|c|c|}
\hline & \multicolumn{2}{|c|}{ Point-mass load } & \multicolumn{5}{|c|}{ Rigid body load } \\
\hline & $n$ quadrotors & 1 quadrotor & $n$ quadrotors & & & quadrotors & \\
\hline Independent DOF & $\begin{array}{c}x_{L} \in \mathbb{R}^{3} \\
q_{1}, q_{2}, \cdots, q_{n} \in S^{2} \\
R_{1}, R_{2}, \cdots, R_{n} \in S O(3)\end{array}$ & $\begin{array}{c}x_{L} \in \mathbb{R}^{3} \\
q \in S^{2} \\
R \in S O(3)\end{array}$ & $\begin{array}{r}\left(x_{L}, R_{L}\right) \in S E(3) \\
q_{1}, q_{2}, \cdots, q_{n} \in S^{2} \\
R_{1}, R_{2}, \cdots, R_{n} \in S O\end{array}$ & & $\begin{array}{r}\left(x_{L}\right. \\
q \\
R_{1}\end{array}$ & $\begin{array}{l}\left.R_{L}\right) \in S E(3) \\
q_{2}, q_{3} \in S^{2} \\
q_{2}, R_{3} \in S O(3)\end{array}$ & \\
\hline $\begin{array}{r}\text { No. of DOF } \\
\text { No. of Actuators } \\
\text { Underactuation }\end{array}$ & $\begin{array}{c}5 n+3 \\
4 n \\
n+3\end{array}$ & $\begin{array}{l}8 \\
4 \\
4\end{array}$ & $\begin{array}{c}5 n+6 \\
4 n \\
n+6\end{array}$ & & & $\begin{array}{c}21 \\
12 \\
9\end{array}$ & \\
\hline Flat outputs & $\begin{array}{c}\mathcal{Y}_{n}=\left[\begin{array}{c}x_{L} \in \mathbb{R}^{3} \\
T_{i} q_{i} \in \mathbb{R}^{3} \\
\psi_{k} \in \mathbb{R}\end{array}\right], \\
i \in\{2, \cdots, n\}, \\
k \in\{1, \cdots, n\}\end{array}$ & $\mathcal{Y}_{1}=\left[\begin{array}{rl}x_{L} & \in \mathbb{R}^{3} \\
\psi & \in \mathbb{R}\end{array}\right]$ & $\begin{array}{c}\mathcal{Y}_{n}=\left[\begin{array}{c}x_{L} \in \mathbb{R}^{3} \\
R_{L} \in S O(3) \\
\Lambda \in \mathbb{R}^{3 n-6} \\
\psi_{k} \in \mathbb{R}\end{array}\right] \\
k \in\{1, \cdots, n\}\end{array}$ & , & $\mathcal{Y}_{3}=$ & $\begin{array}{c}x_{L} \in \mathbb{R}^{3} \\
R_{L} \in S O(3) \\
T_{12} \in \mathbb{R} \\
T_{13} \in \mathbb{R} \\
T_{23} \in \mathbb{R} \\
\psi_{k} \in \mathbb{R} \\
\in\{1,2,3\} \\
\end{array}$ & , \\
\hline No. of Flat outputs & $4 n$ & 4 & & & & 12 & \\
\hline
\end{tabular}

TABLE II: Comparison between multiple cases of quadrotors transporting a suspended load.

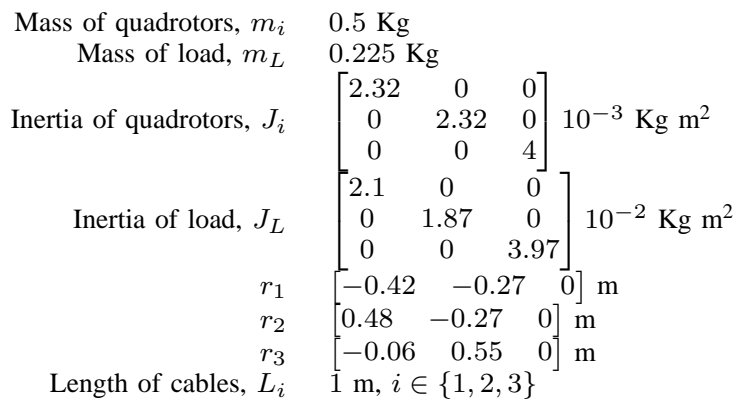

TABLE III: Parameters for simulation and experiments.

For dynamic trajectory generation and for numerical simulation, we consider the system with properties given in Table III. corresponding to our experimental system in Section IV-B.

From the choice of the flat output trajectories for the tension vector, specifically (28), (29), we note that $q_{1}$ is constant, i.e., the unit-vector from the $1^{\text {st }}$ quadrotor to the load attachment point does not change with time, irrespective of the load trajectory. Similarly, from (30), $q_{2}$ can only vary in the $x-z$ plane. However, from (26), since the load trajectory has no motion along $x$, this variation is minimal. This leaves $q_{3}$ free to vary depending on the load trajectory. Figure 5 illustrates how the trajectory of Quadrotor 3 changes for different discrete frequencies of the load trajectory specified in (26). At slower frequencies, the quadrotor trajectory mimics that of the load, albeit with an offset, but as the frequency increases, the trajectory of the quadrotor dramatically changes. Figure 5a illustrates the trajectory of Quadrotor 3 at discrete frequencies of the load trajectory whose time periods vary from $T_{1}=10$ $\mathrm{s}$ to $T_{2}=3.5 \mathrm{~s}$. Figure $5 \mathrm{~b}$ illustrates this for time periods varying from $T_{2}$ to $T_{3}=1 \mathrm{~s}$. Figure 6 illustrates snapshots of a simulation of the system for $T=10 \mathrm{~s}$, and for $T=3.5$ s. Note that at the faster frequency, Quadrotor 3 (red) has a significantly different motion than before to ensure that the load moves faster to track the higher frequency trajectory.

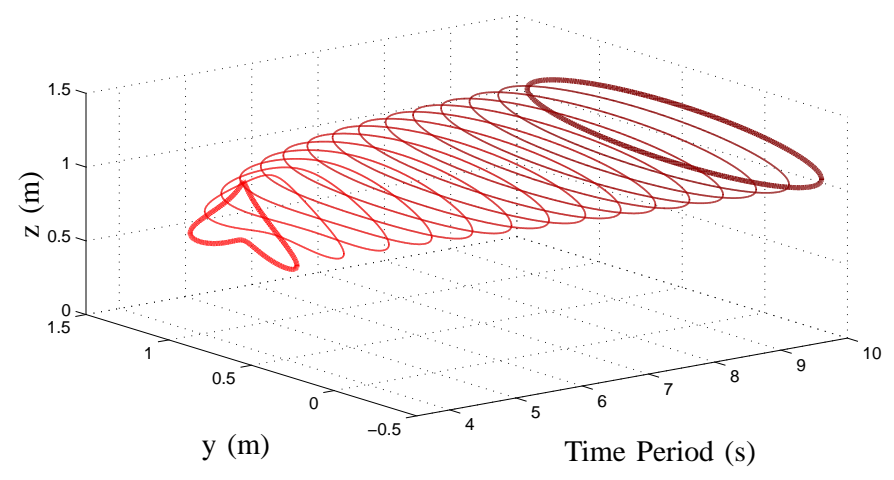

(a)

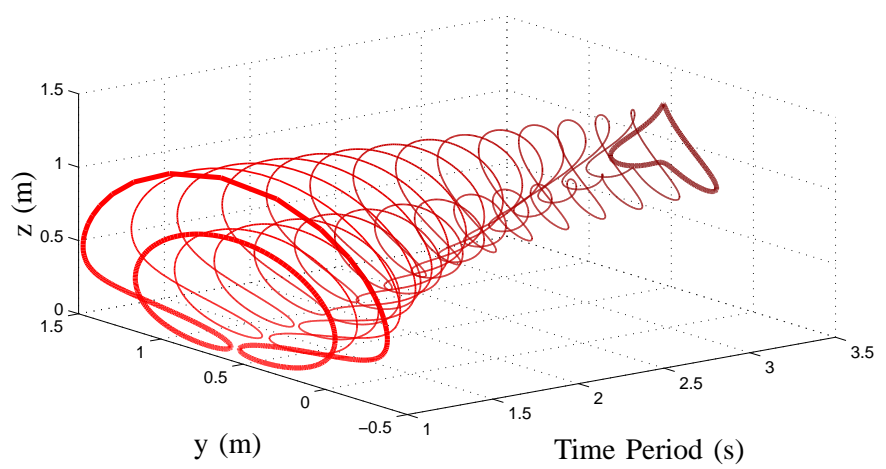

(b)

Fig. 5: Trajectories for Quadrotor 3 as the time period of load oscillation is varied from 10 seconds to 1 second. The first figure shows the variation between 10 and 3.5 seconds, which is used in experiments later on, and the second figure shows the variation from 3.5 to 1 second. Note that the the trajectories become far more aggressive as the desired load trajectory time period goes from 10 seconds to 1 second.

\section{B. Experimental Results}

To illustrate the validity of the proposed method of planning dynamic trajectories, we consider an experimental system of quadrotors (the Hummingbird by Ascending Technologies) and a suspended rigid-body load, such as the one shown in Figure 8. The parameters for this system are the as given in Table III] We will consider the dynamic motion as prescribed 


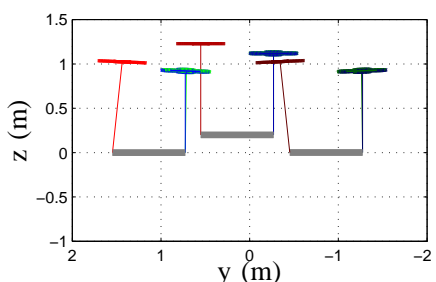

(a)

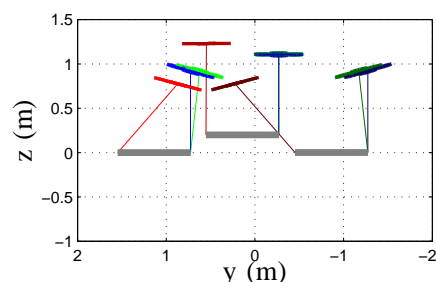

(b)
Fig. 6: Stick figure illustration of simulation of the 3-quadrotor cable-suspended rigid-body load for the cases of (a) $T=10$ s, (b) $T=3.5 \mathrm{~s}$. Note the aggressive trajectory for Quadrotor 3 (red), and the orientations of the other two quadrotors for the higher frequency load trajectory case. A time trajectory of Quadrotor 3 for these two cases is shown on the extremes (bright red) of the plot in Figure 5a.

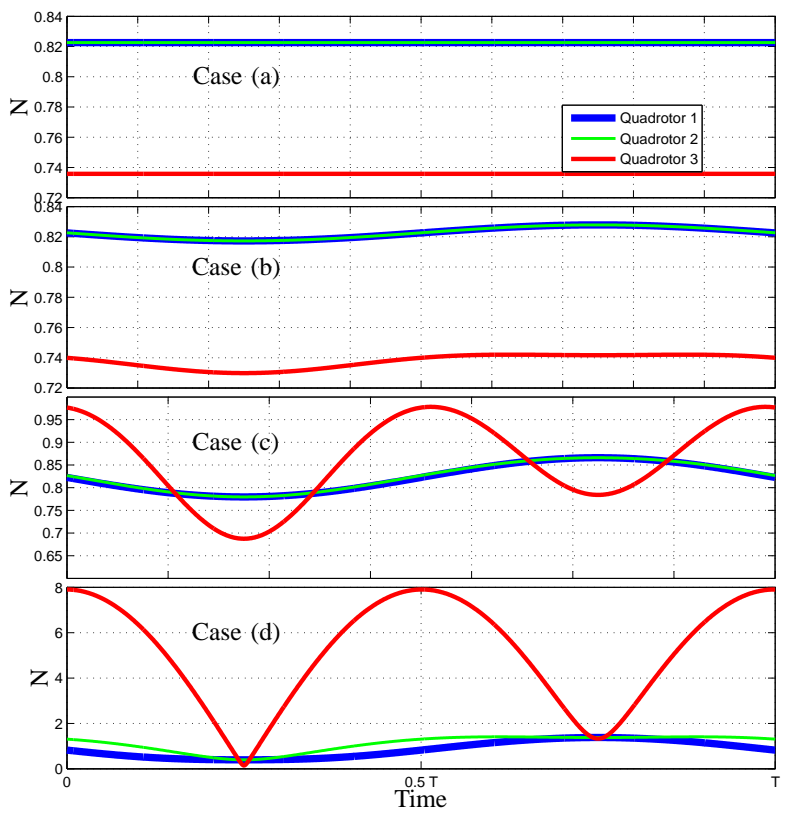

Fig. 7: Tensions in the cables attached to the quadrotors as computed from the differential-flatness for the case of trajectory generation for the cases (a) quasi-static model, and dynamic model for (b) $T=10 \mathrm{~s}$, (c) $T=3.5 \mathrm{~s}$, and (d) $T=1 \mathrm{~s}$. Note that the cable tensions in all cases is positive. Moreover, for the fast dynamic load trajectory, the peak tension in Quadrotor 3 cable is ten times higher than for the slower load trajectory cases.

the flat outputs in (26)-(31). However, instead of the load trajectory being at a discrete frequency, we will consider the load trajectory smoothly increasing in frequency from $f_{1}$ to $f_{2} \mathrm{~Hz}$ in $T$ seconds. Such a trajectory is given by,

$$
x_{L}(t)=\left[\begin{array}{c}
0 \\
A_{y} \cos \left(2 \pi\left((1-\alpha) f_{1}+\alpha f_{2}\right) t\right) \\
A_{z} \sin \left(2 \pi\left((1-\alpha) f_{1}+\alpha f_{2}\right) t\right)
\end{array}\right], 0 \leq t \leq T,
$$

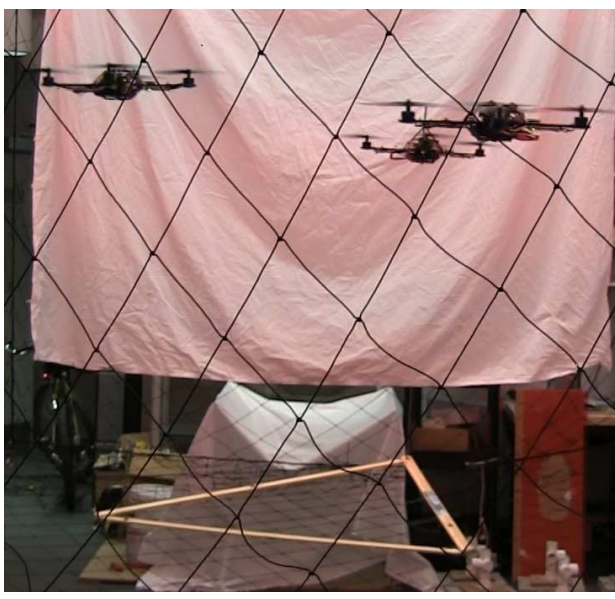

Fig. 8: A snapshot of the experimental setup of the three quadrotors carrying a load. Various parameters for this setup are enumerated in Table III] Experimental videos are available at http://youtu.be/-HAPFrfL4o0, http://youtu.be/byL_wfnhrbw

where $\alpha$ is defined as,

$$
\alpha=\frac{t}{2 T} .
$$

Remark 9. This definition of $\alpha$ ensures that the frequency of the load trajectory smoothly changes from $f_{1}$ to $f_{2}$ in $T$ seconds, as $\alpha$ changes from 0 to 0.5 .

Next, we present two experiments for this same desired load trajectory. The first experiment involves trajectory plans for the quadrotors derived from a quasi-static model [4, 5], where the load velocity, acceleration and higher derivatives are assumed to be zero for all time. This results in a trajectory for the quadrotors that has the same shape as the load trajectory, although its spatially shifted. The second experiment involves trajectory plans derived from a dynamic model, where the quadrotor trajectories are computed using the differential flatness presented in Section $\Pi$

We consider the load-trajectory smoothly varying from $f_{1}=1 / T_{1}$ to $f_{2}=1 / T_{2}$ in $T$ seconds, with $T_{1}=10 \mathrm{~s}$, $T_{2}=3.5 \mathrm{~s}$ and $T=120 \mathrm{~s}$. This choice ensures that the obtained trajectory is within the sensor and actuator limitations of the experimental system. In the experiments, the planned trajectories for the quasi-static and dynamic models serve as inputs to a quadrotor position controller based on [12]. The experiments are carried out with position and orientation feedback for the quadrotors from a Vicon motion capture system. The load position is also tracked through the motion capture system, however it is not used in feedback control. Figure 9 illustrates the performance of the two experiments by comparing the error in tracking the desired load trajectory. In both experiments, the error increases with frequency. However, the load tracking error is about $300 \%-400 \%$ lower in the dynamic case. In the quasi-static case, there is a big phase difference in tracking the load trajectory, leading to large errors. Moreover, in the dynamic case, the tracking error can 


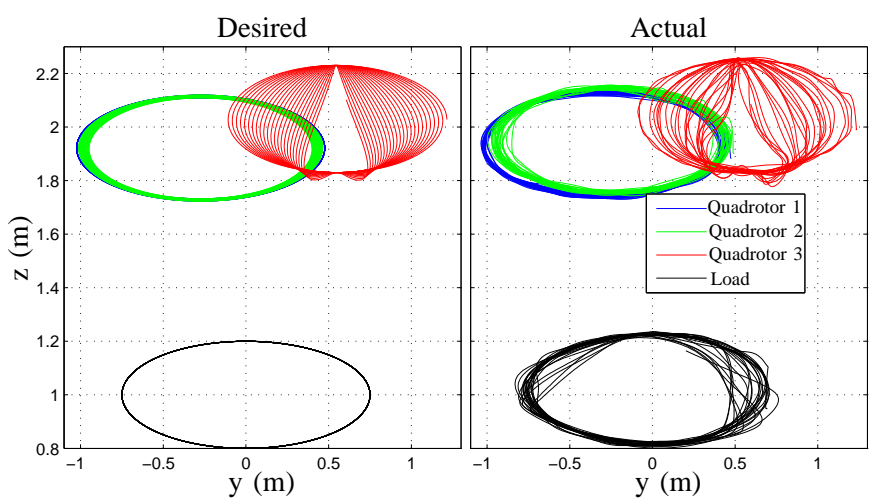

Fig. 10: Desired and actual trajectories from the experiment with trajectory plans derived from a dynamic model.

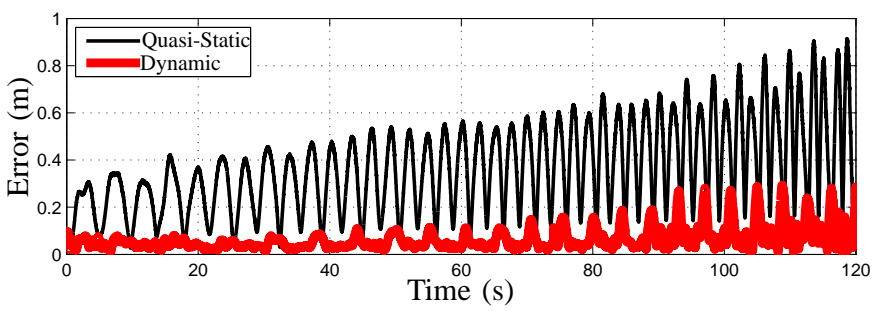

Fig. 9: Norm of the error between the desired and actual load trajectories for the two experiments with trajectory plans generated for quasi-static and dynamic models respectively. The frequency of the desired load trajectory increases with time.

be further reduced by using feedforward moments for the quadrotor that are computed from the differential flatness. For even better load tracking, we will need to make use of the load position as feedback by designing a controller that tracks the position and orientation of the load. This is beyond the scope of this paper, and will be carried out in the near future.

Figure 10 illustrates the desired and experimentally realized trajectories for the three quadrotors and the load for the experiment with trajectory plans derived from a dynamic model. Note that the Quadrotor 3 trajectory dramatically changes as the frequency of the desired load trajectory increases, such that the load moves faster to track the higher frequency trajectory.

\section{CONClusion}

We have addressed the problem of dynamic cooperative transportation of a cable-suspended payload by multiple quadrotors. This is the first study of the entire dynamical problem that simultaneously addresses the problems of underactuation and unilateral constraints, resulting in the development of a general and comprehensive framework for planning dynamically feasible trajectories for the multiple quadrotor point-mass and rigid-body payload system. In particular, we have established that both these systems are differentially flat, and moreover are also differentially-flat hybrid systems, enabling planning of dynamically feasible trajectories for the payload under the cases of positive tensions and also non negative tensions (allowing for zero tensions) in the cables. We presented numerical and experimental results illustrating the superior performance of this method, compared to earlier methods based on quasi-static models. Future work will be directed towards designing controllers that use the load position and orientation as feedback to further improve performance.

\section{ACKNOWLEDGMENTS}

This work was supported by ONR Grant N00014-07-10829, ONR MURI Grant N00014-08-1-0696, NSF IUCRC, and ARL Grant W911NF-08-2-0004.

\section{REFERENCES}

[1] V. Kumar and N. Michael, "Opportunities and challenges with autonomous micro aerial vehicles," Int. J. Robot. Res., vol. 31, no. 11, pp. 1279-1291, Aug. 2012.

[2] R. M. Murray, "Trajectory Generation for a Towed Cable System Using Differential Flatness," in IFAC World Congress, San Francisco, CA, July 1996.

[3] K. Sreenath, N. Michael, and V. Kumar, "Trajectory generation and control of a quadrotor with a cablesuspended load - a differentially-flat hybrid system," in ICRA, Karlsruhe, Germany, May 2013, pp. 4873-4880.

[4] J. Fink, N. Michael, S. Kim, and V. Kumar, "Planning and control for cooperative manipulation and transportation with aerial robots," Int. J. Robot. Res., vol. 30, no. 3, pp. 324-334, Sept. 2010.

[5] N. Michael, J. Fink, and V. Kumar, "Cooperative manipulation and transportation with aerial robots," Auton. Robot., vol. 30, no. 1, pp. 73-86, Sept. 2011.

[6] M. Bernard, K. Kondak, I. Maza, and A. Ollero, "Autonomous transportation and deployment with aerial robots for search and rescue missions," J. Field Robot., vol. 28, no. 6, pp. 914-931, 2011.

[7] I. Maza, K. Kondak, M. Bernard, and A. Ollero, "MultiUAV Cooperation and Control for Load Transportation and Deployment," J. Intell. Robot. Syst., vol. 57, no. 1-4, pp. 417-449, Aug. 2010.

[8] M. Fliess, J. Lévine, P. Martin, and P. Rouchon, "Flatness and defect of non-linear systems: introductory theory and examples," Int. J. Control, vol. 61, pp. 1327-1361, 1995.

[9] R. M. Murray, M. Rathinam, and W. Sluis, "Differential Flatness of Mechanical Control Systems : A Catalog of Prototype Systems," in ASME Int. Mech. Eng. Congress, 1995, pp. 1-9.

[10] D. Mellinger and V. Kumar, "Minimum snap trajectory generation and control for quadrotors," in ICRA, May 2011, pp. 2520-2525.

[11] R. Goebel, R. G. Sanfelice, and A. R. Teel, "Hybrid dynamical systems," IEEE Control Syst. Mag., vol. 29, no. 2, pp. 28-93, April 2009.

[12] T. Lee, M. Leok, and N. H. McClamroch, "Geometric Tracking Control of a Quadrotor UAV on SE ( 3 )," in CDC, Atlanta, GA, 2010, pp. 5420-5425. 